\title{
Le développement des groupes coopératifs agricoles depuis un demi-siècle
}

À la recherche d'un nouveau paradigme

\section{The Development of Agricultural Cooperative Groups in the} Past Fifty Years

Searching for a New Paradigm

\section{Serge Koulytchizky et René Mauget}

\section{Numéro 287, février 2003}

Colloque de l'ADDES: les groupes d'économie sociale

ADDES Symposium: Social-Economy Groups

URI : https://id.erudit.org/iderudit/1022208ar

DOI : https://doi.org/10.7202/1022208ar

\section{Aller au sommaire du numéro}

\section{Éditeur(s)}

Institut de l'économie sociale (IES)

\section{ISSN}

1626-1682 (imprimé)

2261-2599 (numérique)

Découvrir la revue

Citer cet article

Koulytchizky, S. \& Mauget, R. (2003). Le développement des groupes coopératifs agricoles depuis un demi-siècle : à la recherche d'un nouveau paradigme. Revue internationale de l'économie sociale, (287), 14-40.

https://doi.org/10.7202/1022208ar

\section{Résumé de l'article}

À l'aube du XXI ${ }^{\mathrm{e}}$ siècle, on observe des groupes coopératifs complexes que cet article s'attache à décrypter. Ces ensembles ne sont plus tout à fait de même nature que les coopératives de village de la première moitié $\mathrm{du} \mathrm{XX}^{\mathrm{e}}$ siècle. En effet, la globalisation des marchés a entraîné une concentration importante des concurrents, des fournisseurs et des clients des coopératives. Ces dernières ont dû s'adapter et se transformer. Aujourd'hui, les groupes coopératifs conduisent leurs stratégies dans des réseaux complexes d'alliances, entre eux, mais aussi avec des partenaires capitalistes. Les modèles d'analyse présentés durant le siècle dernier n'expliquent plus de façon satisfaisante les mutations en cours : à côté de la valeur de la coopérative pour l'usager apparaît la valeur de la coopérative pour l'actionnaire; l'équité a remplacé l'égalité entre les associés coopérateurs; les principes des pionniers de Rochdale sont bousculés, voire non pertinents pour certains d'entre eux dans l'environnement concurrentiel actuel. Les auteurs cherchent donc à redonner un sens à cette nouvelle race d'organisation qui ne peut pas non plus être expliquée en totalité par les modèles de l'entreprise capitaliste. Dans le modèle de la performance globale de l'entreprise coopérative qui est proposé, la taille de la coopérative ou ses réseaux d'alliances ne sont pas obligatoirement les éléments déterminants du chemin vertueux. La « bonne gouvernance » des groupes coopératifs passe par la quête de sens et la qualité des hommes, mais la multiplicité des parties prenantes complexifie cette gouvernance. 


\section{LE DÉVELOPPEMENT DES GROUPES COOPÉRATIFS AGRICOLES DEPUIS UN DEMI-SIĖCLE ${ }^{(*)}$}

A la recherche d'un nouveau paradigme

(*) Cet article constitue une syn thèse partielle du dossier présenté et remis au colloque Addes 2002 (65 pages).

(**) Addes-Ciriec; maître de confé rences des universités.

$(* \star *)$ Addes-Ciriec ; professeur Essec. par Serge Koulytchizky ${ }^{(*)}$ et René Mauget ${ }^{(* *)}$

A l'aube du XXI siècle, on observe des groupes coopératifs complexes que cet article s'attache à décrypter. Ces ensembles ne sont plus tout à fait de même nature que les coopératives de village de la première moitié du XX siècle. En effet, la globalisation des marchés a entraîné une concentration importante des concurrents, des fournisseurs et des clients des coopératives. Ces dernières ont dîu s'adapter et se transformer. Aujourd'hui, les groupes coopératifs conduisent leurs stratégies dans des réseaux complexes d'alliances, entre eux, mais aussi avec des partenaires capitalistes. Les modèles d'analyse présentés durant le siècle dernier n'expliquent plus de façon satisfaisante les mutations en cours: à côté de la valeur de la coopérative pour l'usager apparaît la valeur de la coopérative pour l'actionnaire; l'équité a remplacé l'égalité entre les associés coopérateurs; les principes des pionniers de Rochdale sont bousculés, voire non pertinents pour certains d'entre eux dans l'environnement concurrentiel actuel. Les auteurs cherchent donc à redonner un sens à cette nouvelle race d'organisation qui ne peut pas non plus être expliquée en totalité par les modèles de l'entreprise capitaliste. Dans le modèle de la performance globale de l'entreprise coopérative qui est proposé, la taille de la coopérative ou ses réseaux d'alliances ne sont pas obligatoirement les éléments déterminants du chemin vertueux. La "bonne gouvernance " des groupes coopératifs passe par la quête de sens et la qualité des hommes, mais la multiplicité des parties prenantes complexifie cette gouvernance.

\section{Introduction générale - Pertinence du modèle coopératif agricole}

L'entreprise coopérative agricole française apparait comme particulièrement marquante dans l'ensemble des autres familles de la coopération et de l'économie sociale, bien que toutes soient porteuses d'un message doctrinalement identique (OIT, ACI). C'est moins par ce message que la coopérative agricole joue actuellement " dans la cour des grands » que par sa masse conséquente, par l'étendue de son domaine historique à la fois patrimonial et culturel; cela s'écrit dans les statistiques, dans les parts de marchés et dans le PIB. 
(1) Les holdings coopératifs, éd. De Boeck (Belgique, 2001) pour le compte du Ciriec intemational.
Ce domaine, cette masse, induit un modèle d' " alliance agricole ", dont la coopération est le pivot (CNMCCA) et qui ne connaît pas d'équivalent dans les autres secteurs de l'économie industrielle; l'émergence d'une couche sociale spécifique, celle des "notables " du monde paysan, n'était pas prévue par la doctrine, mais elle a durablement marqué - positivement en général - le siècle dernier. Ce sont ces mêmes personnes qui ont porté plus ou moins volontairement l'élargissement de la structure de la coopérative-mère au groupe coopératif, le passage d'une logique paysanne à une logique industrielle et commerciale et plus tard financière.

Une autre force du mouvement réside dans son enracinement dans le territoire dont les administrateurs demeurent les garants. Cet enracinement historique est précieux. Il donne un avantage (coopératif) évident aux groupes qui savent l'utiliser. Parfois considéré comme un handicap, l'enracinement local est le premier maillon d'une quête de sens fondée sur le pragmatisme et non sur les idéologies.

Cette même recherche est menée en parallèle par certaines entreprises capitalistes dites " citoyennes » qui visent une légitimité autre que celle qui découle de la seule propriété (property rights).

Dommage que dans l'échange - lorsqu'il y a échange - ce soit le modèle coopératif qui apparaisse redevable de leçons d'efficacité et que l'on ne sache pas promouvoir son antériorité et sa pertinence dans la présentation d'une mission plus large de l'efficience des organisations productives.

C'est ce que les auteurs tentent modestement de corriger ici en présentant un modèle coopératif "en mouvement ", qui au fil des mutations de structures des cinquante dernières années a parfois été donné comme généralement peu lisible et porteur d'un message brouillé, voire dépassé. De la coopérative de village au groupe coopératif multinational, c'est la mutation quantitative et qualitative que nous avons vécue. Cette mutation a le plus souvent été la réponse aux évolutions de l'environnement concurrentiel et politique. Les groupes coopératifs qui en résultent doivent être analysés avec de nouvelles grilles de lecture. La distinction ancienne entre "capitalisme " et " acapitalisme " n'est plus pertinente dans les groupes complexes. L'apport de valeur par la coopérative pour l'usager peut cohabiter avec la valeur pour l'" actionnaire " (retombées de dividendes) lorsqu'il y a partenariat avec le secteur capitaliste ou délocalisation partielle de productions agricoles. Cela a été développé dans l'ouvrage Les holdings coopératifs, évolution ou transformation défnitive, piloté par Daniel Côté(1) [voir notamment l'article de Serge Koulytchizky et René Mauget].

Il importe maintenant de tracer une convergence entre un "modèle d'affaires » rendu indispensable par l'exacerbation de la compétition et un «modèle coopératif vertueux ». Contre le grand écart attendu, la construction d'une "nouvelle alliance " est le challenge qui s'impose au début du siècle. L'idée d'un «renouveau coopératif » passe par la recherche d'un nouveau paradigme. 
(2) Cette prenière partie s'appuie sur le travail effectué par René Mauget et Serge Koulytchizky pour le collogue de Maraussan 2001.
Notre contribution comporte trois parties. La première consistera à analyser le développement quantitatif et qualitatif des coopératives agricoles au cours du XX siècle qui conduit aujourd'hui à des groupes complexes ${ }^{(2)}$. La deuxième partie, à partir d'exemples de groupes coopératifs, présentera la nature de ces mutations. Enfin, nous chercherons les bases d'un nouveau modèle d'analyse pour ces groupes complexes.

\section{Evolution des coopératives durant le XX ${ }^{\mathrm{e}}$ SIÈCLE}

(3) Etude René Mauget et Valérie Dulawa, Essec, Imia, 2001.

\section{Evolution quantitative des coopératives agricoles durant le $\mathrm{xx}^{\mathrm{e}}$ siècle}

\section{Remarques préalables}

Il nous a été difficile de retrouver des statistiques précises avant 1965, si ce n'est quelques chiffres avant la guerre de 1939. Ainsi, nous possédons des séries statistiques depuis trente-cinq ans. Cependant, les informations statistiques du SCEES qui recouvrent le champ juridique des coopératives, unions de coopératives et Sica ne représentent plus la réalité des groupes coopératifs filialisés. Dans ce contexte, les fédérations de coopératives sortent des informations statistiques approximatives qui intègrent dans leur périmètre les filiales. Nous utiliserons ces deux types d'informations.

\section{Le poids des coopératives dans les pays de l'Union européenne (3)}

Les annexes 1 et 2 nous montrent une forte disparité du poids des coopératives dans l'économie agro-alimentaire de chaque pays. Les pays du nord de l'Europe, hormis le Royaume-Uni, sont fortement impactés par les coopératives. Les pays du sud de l'Europe possèdent un secteur peu important en parts de marché. La France et l'Allemagne se situent à un niveau intermédiaire. Ainsi, la France représente un peu plus de $20 \%$ du CA de l'agriculture et des IAA de l'UE et $30 \%$ du CA des coopératives de l'UE.

L'évolution quantitative des coopératives agricoles en France sur trente-cinq ans

(Voir tableau 1 et annexe 3.)

On note:

- une diminution de $40 \%$ du nombre d'entreprises coopératives sur trentecinq ans, mais, si l'on calcule le nombre de centres de décision stratégique, cette baisse serait beaucoup plus importante, car une partie non négligeable de la concentration a été effectuée sous forme d'unions (par exemple Sodiaal, Epi Centre, etc.); dans ce cas, les coopératives de base sont toujours dans les statistiques. Le nombre des filiales a au contraire augmenté: 1500 en 2001. 
-Le chiffre d'affaires a été multiplié par plus de vingt en francs courants, ce qui correspond à un multiple de 3 en francs constants depuis 1965. Il atteint 426 milliards de francs en 2001 (soit 65 milliards d'euros dont 20 dans les filiales).

- Neuf agriculteurs sur dix adhèrent à au moins une coopérative; ce chiffre évolue peu sur trente-cinq ans.

- Le nombre de salariés a augmenté sensiblement pour passer de 75000 à 120000 environ en 2001.

Les coopératives sont plus présentes dans la collecte et la première transformation que dans les activités proches du consommateur final. On note corrélativement, par rapport aux entreprises capitalistes, une plus faible valeur ajoutée. Ce handicap se retrouve en moyenne sur les autres indicateurs (EBE, CAF, résultat avant impôt).

En 2001, les coopératives agricoles maitrisent $57 \%$ de la collecte, $35 \%$ de la transformation et $60 \%$ de l'approvisionnement. Ces chiffres constituent des moyennes avec de fortes disparités.

Cependant, en dehors d'une dizaine de groupes, les coopératives sont peu présentes, en bonne position, au sein des oligopoles agro-alimentaires, ce qui explique leur infériorité relative en matière de performances économiques à partir des indicateurs classiques. On pourrait également s'interroger sur la pertinence de ces indicateurs pour les coopératives agricoles en fonction de leurs finalités.

\section{Tableau 1}

Evolution quantitative des coopératives depuis trente-cinq ans

\begin{tabular}{|c|c|c|c|c|c|c|}
\hline Années & 1929 & 1939 & 1965 & 1972 & 1978 & $2001^{(1)}$ \\
\hline $\begin{array}{l}\mathrm{Nb} \\
\text { d'organismes }\end{array}$ & $16082^{(3)}$ & 22886 & 5717 & 4700 & 4141 & 3500 \\
\hline $\begin{array}{l}\text { CA milliards } \\
\text { de francs }\end{array}$ & & & 20,6 & 50 & 110 & $\begin{array}{c}426^{(2)} \\
65 \text { milliards } \\
\text { d'euros }\end{array}$ \\
\hline Salariés & & & 80211 & 95700 & 107000 & $120000^{(4)}$ \\
\hline Source & $C I A$ & $B I T$ & SCEES & SCEES & SCEES & CFCA \\
\hline
\end{tabular}

(1) Hors Cuma, 13300 en 2001.

(2) Le SCEES donnait en 200145 milliards d'euros soit 295 milliards de francs de CA et 62000 salariés. En effet, les statistiques du SCEES ne prennent pas en compte les filiales.

(3) Dont 13312 syndicats ayant une activité de commerce et 2770 coopératives dont 2526 laiteries.

(4) En 2001, la CFCA donne 150000 salariés permanents (voir notions de permanents et d'équivalents temps plein).

Evolution du périmètre coopératif (Source CFCA)

Le périmètre a globalement augmenté jusqu'à la fin des années 80 (voir les facteurs de développement du secteur coopératif). 
(4) Etude de René Mauget sur les restructurations dans les IAA, 1996-2000 (avec Francis Declerck).

(5) Voir texte développé au col loque Addes 2002.
A la fin des années 80 et au début des années 90 , ce solde a régressé suite à un désengagement souvent forcé de plusieurs grands groupes (ULN, Arcadie, La Brie, Gouessant France-Volailles, Bressor, Unicolait, Transagra...). La période 1994-1996 a "marqué " le retour à l'équilibre du périmètre et, depuis 1997, selon les sources CFCA, ce périmètre s'accroît de façon significative, soit de 3,8 milliards d'euros (25 milliards de francs) entre 1997 et 2002. Sur six ans, cela représente $3 \%$ environ du chiffre d'affaires des IAA.

On note donc que, dans un contexte difficile et dans un marché en voie de globalisation, les coopératives françaises résistent bien après quelques revers souvent consécutifs à des imprudences stratégiques dans un contexte financier d'argent cher.

Les restructurations, ces dernières années, sont surtout la résultante de stratégies d'alliances entre coopératives (union, SA, fusion). Durant ces trois dernières années, plus de $50 \%$ des opérations de restructuration ont été effectuées entre coopératives, par exemple Cana-Caval (Terrena), Agrial, Sigma-UNCAA (In vivo), Nouricia, Cristal Union, etc.

Ces opérations n'ont pas d'effet direct sur le périmètre, mais ces pôles peuvent constituer une dynamique de restructuration à terme et dégager $\mathrm{du}$ « cash " pour conduire des opérations de croissance externe, par exemple le rachat de Bourgoin par Gastronome (Cana-Caval).

Lextension du périmètre s'est en revanche effectuée par des prises de contrôle d'entreprises capitalistes. A titre d'exemple:

- Champagne céréales et autres coopératives $\rightarrow$ Grands Moulins de Paris (GMP);

- Maïsadour

- Coop de Pau

$\rightarrow$ Delpeyrat;

- Gastronome

- Val d'Orbieu

- SDA

$\rightarrow$ Grimaud et Rougié, Bizac;

$\rightarrow$ BSA (Bourgouin);

$\rightarrow$ Listel, Cordier;

$\rightarrow$ Beghin Say...

L'expansion des coopératives s'est également effectuée à l'étranger (10 à $15 \%$ des opérations des coopératives agro-alimentaires); ceci est nettement moins important que pour les entreprises capitalistes françaises qui, pendant les deux années 1999 et 2000, ont effectué les deux tiers de leurs opérations de rachat à l'étranger ${ }^{(4)}$.

\section{Les facteurs de développement des coopératives agricoles durant le $\mathrm{XX}^{\mathrm{e}}$ siècle ${ }^{(5)}$}

Le système coopératif a connu un développement important en France et, malgré quelques régressions de son périmètre au début des années 90, il poursuit aujourd'hui, au début du second millénaire, sa progression.

Ce développement, différent selon les secteurs et les activités dans un même secteur, est la résultante d'un certain nombre de facteurs que nous pouvons lister : - les pressions économiques diverses et variées selon les périodes (la coopération fille de la nécessité) ;

- les facteurs humains et les mouvements d'idées (notables, curés, instituteurs, plus tard syndicalistes, Jac, etc.); 
- la constitution d'appareils politiques de tendances opposées, « rue d'Athènes ", plutôt à droite et corporatiste, et " boulevard Saint-Germain ", plutôt gauche laïque et républicaine. Puis réunification de ces tendances depuis 1966 avec la création de la CFCA ;

- la constitution de groupes coopératifs comme pôle de restructuration sous forme de fusion ou d'union, aujourd'hui des groupes complexes avec rachat d'entreprises capitalistes ou alliance avec celles-ci (par exemple Sodiaal, Terrena, Euralis, Copagri, Champagne céréales, Epi Centre, Agralis, SDA) ;

- le désengagement du capital agro-alimentaire non coopératif. En effet, dans un univers globalisé, les entreprises capitalistes, pour optimiser la rentabilité des capitaux, sont amenées à changer de secteur ou de zone géographique. Les coopératives ancrées sur le territoire peuvent être conduites à racheter ces activités (Préval, GMP, Beghin Say...) ;

- le soutien législatif et financier de l'Etat. A titre d'exemple : loi de 1884 autorisant la création de syndicat agricole ; loi de 1936 ayant imposé le stockage aux coopératives et à elles seules ; législation sur les Sica et les groupements de producteurs entre 1960 et 1962 ; loi de 1972 (options dérogation à l'exclusivisme et associés non coopérateurs) ; loi de 1991 à dominante financière, même si cette loi n’a pas eu les effets escomptés ; textes sur le crédit agricole et le financement des coopératives (1920). Le facteur législatif a souvent été accompagné par un soutien financier (subventions, prêts bonifiés). Aujourd'hui et au fil des ans, ce soutien s’est atténué.

\section{Développement ou mutation progressive?}

Au cours du siècle et surtout depuis une trentaine d'années, la législation sur les coopératives a évolué vers une plus grande souplesse. Les stratégies et les structures des coopératives ont muté pour s'adapter à l'environnement concurrentiel. Dans ces évolutions, les principes ont été bousculés, notamment l'acapitalisme et l'exclusivisme.

De même, la filialisation dans les groupes coopératifs agro-alimentaires en partenariat avec les entreprises capitalistes a engendré une nouvelle race d'entreprises qui ne peuvent plus se définir selon les principes coopératifs classiques. C'est pour cela qu'aujourd'hui les entreprises coopératives parlent davantage de finalité et de valeurs coopératives que de principes, les valeurs étant définies ainsi : l'équité, qui a remplacé l'égalité dans de nombreuses coopératives; la transparence; la solidarité sélective (parfois). Certains diront que ces valeurs ne sont pas spécifiquement coopératives, mais sont des valeurs citoyennes dans nos démocraties dites avancées.

Les finalités, quant à elles, peuvent également évoluer dans ces groupes filialisés, notamment si le groupe transforme des produits ne provenant pas des adhérents (internationalisation ou multirégionalisation). Dans ce cas, le revenu que l'agriculteur obtient de sa coopérative peut prendre deux aspects: 
(6) « Claude Vienney par lui-même, entretien avec André Chomel et Marie-Claire Malo, 1996 » Coopération et économie sociale au second $x x^{e}$ siècle, Claude Vienney (1929-2001), « Les cahiers de 1'éco nomie sociale $n^{0} 1$, Institut de l'économie sociale-L'Harmattan, Paris, juillet 2002.
- un revenu en tant qu'usager;

- un revenu en tant qu'actionnaire direct ou indirect dans les filiales. Il convient également de signaler que, dans le cadre de groupes complexes filialisés avec partenaires capitalistes, la question fiscale ne se pose guère, puisque le groupe coopératif a été fiscalisé sans changer le statut fiscal de la mère.

Cette première partie montre l'importance de l'impact économique des coopératives agricoles dans l'Union européenne et notamment en France. A l'aube du XXI ${ }^{e}$ siècle, le développement semble se poursuivre dans un contexte de globalisation des marchés et sous l'effet notamment de la délocalisation du capital agro-alimentaire (Beghin Say, GMP, etc.). Les coopératives, dans ce contexte, constituent un atout déterminant pour la pérennité des bassins de productions agricoles.

Cependant, ce développement s'est effectué avec des mutations. La plupart des théories et des modèles d'analyse présentés dans la seconde partie du XX pour expliquer la coopération classique ne sont plus pertinents pour comprendre totalement la logique des groupes coopératifs complexes. Claude Vienney, parlant de transformation des coopératives, dit : "Dans la transformation, il y a aussi des coopératives qui sortent complètement $d u$ champ, qui cessent d'être des coopératives et donc cela devient plus difficile à saisir. Effectivement, il y en a qui naissent, il y en a qui fonctionnent, ily en a qui se transforment et, parmi celles qui se transforment, il y en a qui conservent leurs caractéristiques et il y en a qui les perdent ${ }^{(6)}$. "Pour C. Vienney, l'hybridation dans les groupes coopératifs doit admettre une limite : "Pour moi, dit-il, une caractéristique de l'organisation coopérative, c'est des situations dans lesquelles les gens, pour quelque raison que ce soit, préferent mettre une somme d'argent pour soutenir une activité dont ils ont besoin plutôt que de mettre la même pour que ce soit rentable. Alors aujourd'hui, il est vrai qu'il y a des quantités de situations où on est dans des préférences pour la rentabilité." "

Cette analyse est certes intéressante, mais notre approche sera différente et notamment parce que postérieure. En effet, dans la deuxième partie de cet article, nous allons présenter quelques groupes coopératifs avec leurs particularités et nous tenterons dans une troisième partie de présenter un modèle de synthèse.

\section{Les groupes coopératifs au XXl ${ }^{e}$ siècle}

\section{La notion de groupe coopératif}

Le groupe coopératif selon notre approche est constitué d'une mère ou de plusieurs mères sous statut de coopérative agricole ; il peut y avoir également une union (fille-mère). C'était le cas de l'ULN, cotée en Bourse avec des titres participatifs, la Commission des opérations de Bourse (Cob) consolidant sur cette " fille-mère ». Dans le cas des groupes coopératifs, la structure holding est une fille (voir le schéma en page suivante). 


\section{Le groupe coopératif, schéma générique}

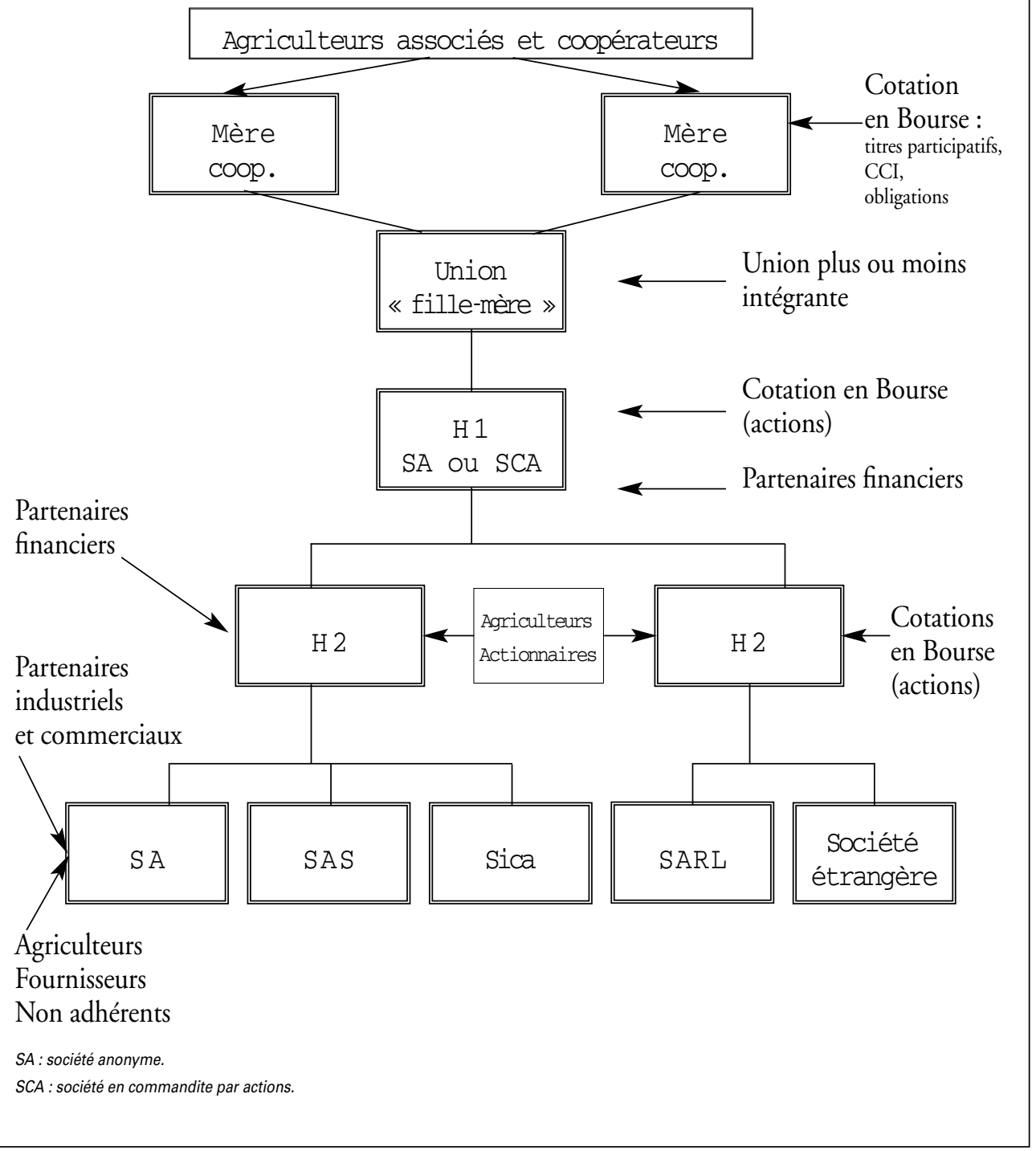

\section{Mutations stratégiques et structurelles dans les groupes coopératifs agro-alimentaires}

L'environnement concurrentiel en 2003 n'est plus celui du début du siècle dernier. Nous sommes passés d'une agriculture relativement protégée avec des petites coopératives de collecte à des marchés agro-alimentaires globalisés avec une baisse tendancielle des protections et une concentration des entreprises situées en amont et en aval. 
(7) Voir contribution de René Mauget et de Michel Forestier au $\mathrm{XV}$ colloque Addes, mars 2000, Recma, $\mathrm{n}^{\circ}$ 278. Voir aussi R. Guillouzo, P. Perrot, Ph. Ruffio, «Stratégie d'alliances et configu rations des groupes coopératifs agricoles 》, Recma, nº 285. (8) En 1997, la consolidation regroupait 82 sociétés pour le groupe Cana.
Dans cet univers, les stratégies de concentration et d'intégration verticale des coopératives ont souvent été une réponse aux évolutions de l'environnement, même s'il existe encore de nombreuses stratégies de niche. Dans ce contexte, les coopératives agricoles existent de moins en moins à l'état pur. Elles constituent des groupes, notamment lorsqu'elles transforment leurs produits, autour d'une coopérative, articulant des filiales de statuts non coopératifs. Les filiales sont en outre souvent détenues en commun avec d'autres coopératives, voire des sociétés capitalistes industrielles ou bancaires. Se trouvent ainsi combinées dans les faits des logiques différentes, coopératives et capitalistes. Pour l'une, le facteur risqué est le produit, pour l'autre, il s'agit du capital ${ }^{(7)}$.

A titre d'exemple, on peut citer quelques chiffres sur la Cana pour montrer ce glissement vers les filiales ${ }^{(8)}$. La filialisation massive a été effectuée en 1989 après l'émission de titres participatifs et l'entrée en Bourse en 1988. En 1997, seulement un tiers du chiffre d'affaires était effectué dans la coopérative, contre les trois quarts en 1985. Il n'y avait plus que $25 \%$ des salariés dans la coopérative, alors qu'en 1985 ils étaient plus des trois quarts.

\section{Pourquoi ces évolutions?}

Avant d'analyser les conséquences, il est intéressant de revenir sur le pourquoi de ces évolutions. Nous avons identifié au moins cinq types de raisons.

- Des raisons stratégiques consécutives à une globalisation des marchés: cette globalisation a presque toujours eu comme conséquence une concentration, notamment commerciale, mais aussi une nécessité de réduire les coûts et (ou) de différencier les produits.

- Des raisons juridiques: contraintes statutaires limitant la capacité commerciale (règles d'exclusivisme et de territorialité, notamment); contraintes pour faire des alliances entre le secteur privé et la société mère coopérative.

- Des raisons financières: difficulté pour mobiliser les capitaux propres avec la société mère, d'où développement des holdings.

- Des raisons fiscales parfois: intérêt de la structure holding pour consolider les résultats des filiales.

- Des raisons de management, de prise de décision et de gestion : simplification, rapidité, meilleure vision des rentabilités par métier, recentrage facilité, etc.

Il convient cependant de noter que la filialisation n'est pas la réponse unique à ces problèmes, ni une panacée. En résumé, le but de cette évolution était et est toujours d'assurer la survie et (ou) d'améliorer l'efficacité du groupe coopératif face à l'accroissement de la dynamique concurrentielle. Aujourd'hui, après une dizaine d'années de filialisation, il est intéressant de s'interroger sur les conséquences de ces mouvements. 
(9) En droit, deux fois le capital social.

(10) Dans la pratique, la loi de 1972 en France permet de reva loriser le capital social avec les réserves disponibles, dans la limite de l'évolution de la rente viagère.

(11) Dans la pratique, la loi de 1972 en France permet de revaloriser le capital social avec les réserves disponibles, dans la limite de l'évolution de la rente viagère

\section{Conséquences de ces mutations}

Les conséquences en matière de rémunération des agriculteurs et de critères d'efficacité

- Dans la coopérative agricole classique

Dans la coopérative agricole classique, le facteur risqué est le produit, alors que dans une entreprise capitaliste, il s'agit du capital. Le capital social dans la coopérative est plus proche d'un capital obligation ou d'un prêt des membres que d'un capital action. Il subit néanmoins le risque de faillite (9). Les implications stratégiques et financières de cette forme d'organisation sont donc déterminantes.

Le capital bénéficie d'une rémunération limitée pouvant aller de 0 jusqu'au taux moyen des obligations (en France). Les sociétaires n'ont pas de droit sur les réserves ${ }^{(10)}$. En revanche, les surplus dégagés par l'exploitation peuvent être distribués aux adhérents sous forme de ristournes au prorata des transactions économiques avec la coopérative. C'est le principe " acapitaliste ». Ainsi, la valeur de la coopérative pour l'adhérent se manifeste d'abord par l'équation: (acompte + complément de prix + ristournes) x quantités produites. A cela on peut ajouter la rémunération du capital social.

L'approche patrimoniale de la coopérative pour l'adhérent, par le bilan, est également plus complexe que dans l'entreprise capitaliste. En effet, l'adhérent n’a pas de droit sur les réserves, il n’a pas droit aux plus-values. La valeur de son capital reste à la valeur nominale comme dans le cas d'un prêt ou d'un compte associé( ${ }^{(1)}$, il n'a pas droit non plus à des dividendes.

- Cas du groupe complexe

Les implications en matière de management stratégique et financier sont importantes pour la coopérative et pour l'agriculteur. En effet, dans un groupe complexe, surtout lorsqu'il y a des partenaires non coopératifs, qu'ils soient financiers (banque) ou industriels, nous avons un mariage entre deux entités qui souhaitent optimiser un facteur risqué différent: les agriculteurs, le produit; le partenaire capitaliste, le capital.

Pour résumer, la rémunération du produit dans cette sphère évolue de la façon suivante:

- Rappel coopérative classique.

Acompte + complément de prix + ristournes.

- Sphère aval dans les groupes complexes (le résultat est externalisé). Prix de marché + dividendes avec possibilité, depuis la loi du 3 janvier 1991 en France, de faire une remontée des dividendes avec avoir fiscal jusqu’aux sociétaires.

Possibilité de plus-value pour l'agriculteur si celui-ci a investi directement dans la filiale (par exemple Maltor).

Il reste que la société mère peut encore effectuer des arbitrages au sein de la coopérative mère entre:

- intérêt sur le capital social,

- dividendes avec avoir fiscal,

- ristournes,

ce que nous retrouvons aujourd'hui dans la pratique de ces groupes. 


\section{Présentation de quelques groupes coopératifs complexes à l'aube du XXI' siècle (schémas simplifiés)}

Ces exemples permettront d'illustrer la nature des changements et les limites des modèles " classiques " pour expliquer cette nouvelle « race » d'organisation hybride.

\section{Exemple Cana (coopérative polyvalente, Ouest) avant création de Terrena}

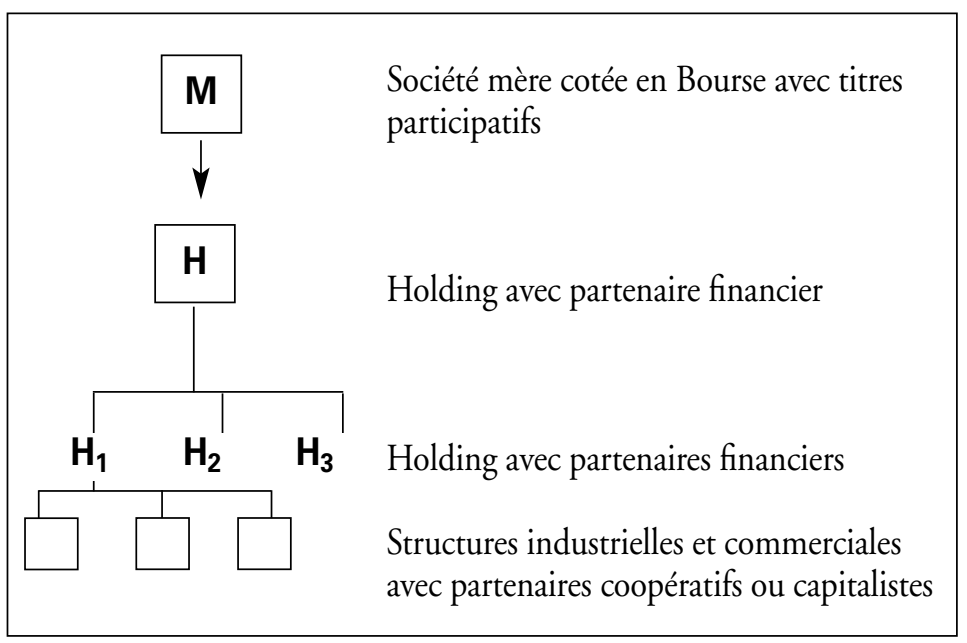

\section{Particularités}

- Cotation en Bourse de la mère coopérative agricole avec titres participatifs.

- Partenaires financiers dans les sociétés holdings.

- Partenaires capitalistes et coopératifs dans les filiales industrielles et commerciales.

- Activités hors zone avec des agriculteurs non adhérents (notamment filière volailles après la reprise partielle du groupe BSA [Bourgoin]).

- Approche multipartenaire.

- Création d'une union 50/50 (Terrena) avec la Caval en 2000 et rattachement des filiales à cette union.

Dans ce modèle, la cotation en Bourse avec des titres participatifs ne pose pas de gros problèmes, car le titre participatif ne donne pas de pouvoir. Il est cependant partiellement lié au résultat du groupe coopératif, donc à la politique de répartition entre les sociétaires (acompte + complément de prix); le résultat affiché peut être modifié pour faire apparaître un résultat plus important.

La présence de partenaires financiers dans les holdings et de partenaires industriels et commerciaux dans les filiales du type SA aura tendance à faire évoluer le prix d'acompte vers un prix de marché; le résultat distribuable peut dans ce cas être réparti sous forme de ristournes ou de dividendes. Un débat peut cependant avoir lieu avec les partenaires industriels et financiers sur la part du résultat des filiales conservée en autofinancement et celle qui sera distribuée aux sociétaires sous forme de ristournes ou de dividendes. 
La présence d'apporteurs non adhérents en dehors de la zone peut également justifier un traitement différencié. Cette question se pose dans presque tous les groupes complexes où cohabitent des agriculteurs adhérents et des agriculteurs fournisseurs ou clients non adhérents au sein de la société mère (option dérogation) ou des filiales.

Exemple Champagne céréales (groupe céréalier, Est)

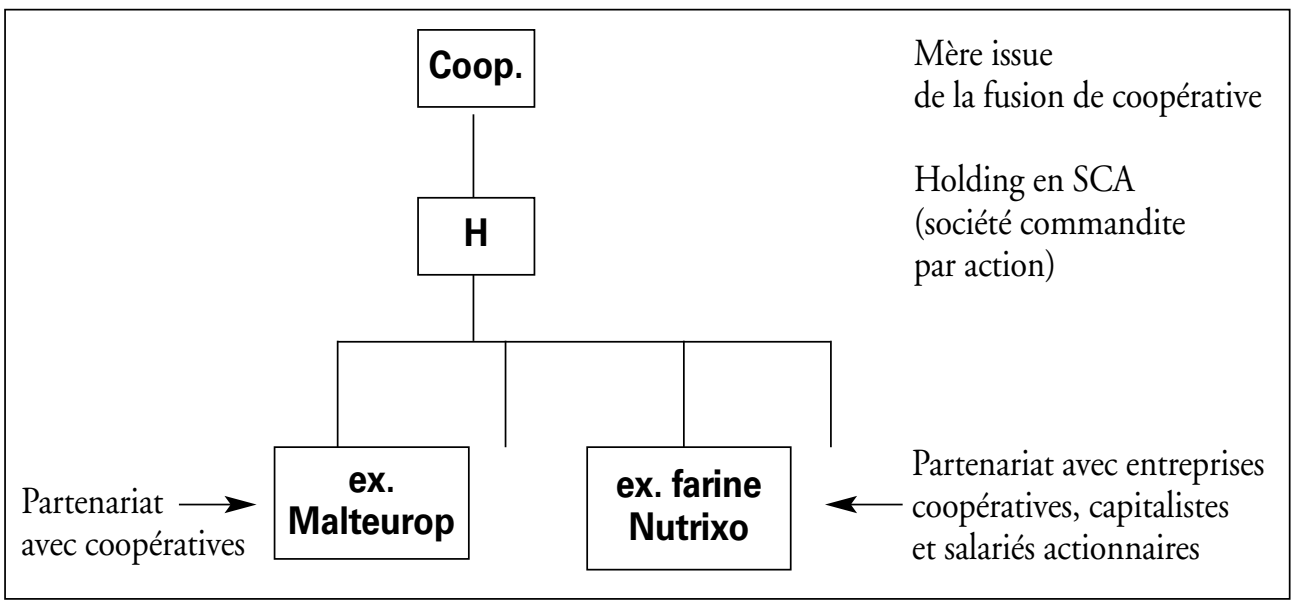

Particularités

- Holding en commandite par action avec partenaires commanditaires.

- Groupe internationalisé; filiale Malteurop présente en Chine, en Argentine, en Ukraine; filiale farine avec partenaires capitalistes et salariés suite à la reprise des Grands Moulins de Paris (GMP).

Dans ce groupe, la redistribution du surplus est complexe: la politique de fixation des prix de la matière première orge (Malteurop) peut être différente selon qu'il s'agit d'agriculteurs sociétaires des coopératives, actionnaires des filiales ou d'agriculteurs seulement fournisseurs (français ou étrangers). Un arbitrage peut aussi être effectué sur le surplus entre: complément de prix, ristournes, dividendes, rémunération du capital social (ces dernières années, Champagne céréales a déjà effectué un " mixte » de distribution entre rémunération du capital social, dividendes et ristournes). Dans le cas des filiales farine et notamment GMP, la politique de distribution peut être rendue plus complexe par la présence d'un actionnariat différent et notamment l'arrivée d'un actionnariat salarié. Nous pouvons ainsi faire entrer en concurrence trois facteurs risqués: le produit, le capital, le travail.

Enfin, la structure en commandite par action peut potentiellement avoir un effet sur cette répartition, ce qui ne semble pas le cas aujourd'hui, car les seuls commanditaires sont à notre connaissance des coopératives voisines n'ayant pas, à l'époque de la fusion, souhaité fusionner tous leurs actifs, mais ayant cependant voulu conserver des parts dans les filiales (exemple Malteurop). 
Cas Epi Centre avec Maltor (groupe céréalier, Centre)

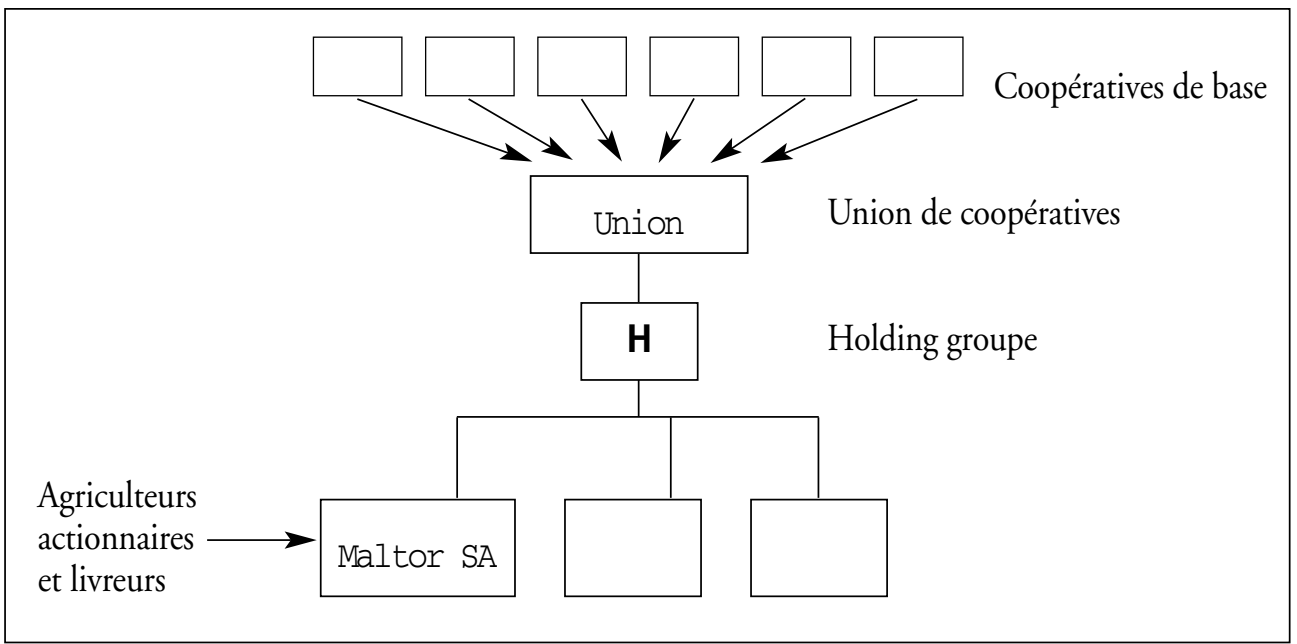

(12) Pas de moins-value, car la coopérative $\mathrm{s}^{\prime}$ engage à reprendre le capital à son nominal dans ce cas de figure.
Dans ce modèle, la particularité est la double participation de l'agriculteur: adhérent de sa coopérative et actionnaire direct de sa filiale. Cette double participation financière peut conduire l'adhérent actionnaire de la filiale (il pourrait également être non adhérent, mais seulement actionnaire apporteur) à un comportement différent, voire plus patrimonial, car en cas de retrait de la filiale, l'agriculteur a droit aux plus-values, ce qui n'est pas possible dans la coopérative.

Cet exemple nous conduit donc à une nouvelle évolution dans la dynamique de redistribution potentielle du surplus pour l'agriculteur par rapport aux deux cas précédents. En effet, nous pouvons jouer sur les variables de distribution suivantes: acompte; compléments de prix; ristournes; dividendes au prorata du capital social détenu dans la coopérative; dividendes au prorata du capital détenu dans la filiale; intérêt sur capital social dans la coopérative; plus-values en cas de retrait de la filiale ${ }^{(12)}$.

Les autres partenaires capitalistes peuvent bien sûr intervenir dans la décision du « mixage ».

Exemple Limagrain (groupe semencier, Massif central)

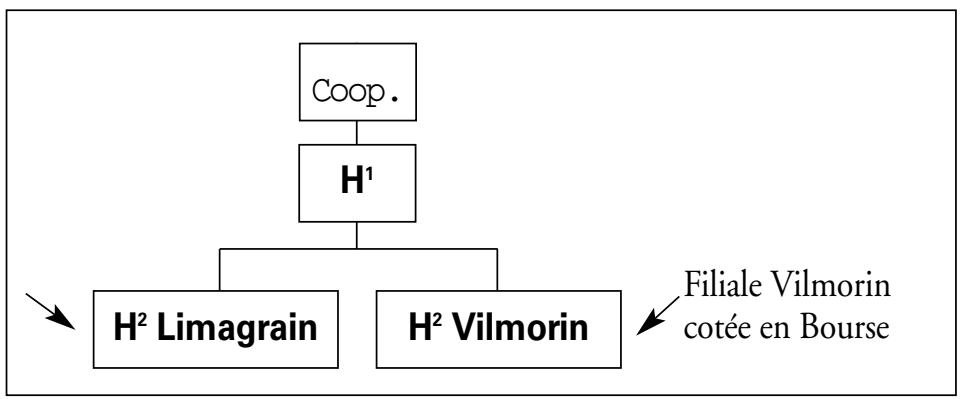




\section{Particularités}

- Groupe très internationalisé qui possédait avant la loi du 3 janvier 1991 une formule de société en nue-propriété pour faire remonter les profits des filiales étrangères jusqu'aux sociétaires.

- Cotation en Bourse d'une filiale résultant d'une opération de croissance externe dans un domaine d'activité de diversification par rapport à l'activité principale de départ.

Nous notons ce cas parce qu'il s'agit d'une première dans le milieu coopératif agricole français. Plusieurs groupes coopératifs français ont des projets de cotation.

Dans le cas de Limagrain, il s'agit d'une filiale de diversification, donc l'enjeu est important, mais pas aussi déterminant que pourrait l'être une cotation en Bourse de la structure holding de tête d'une coopérative (type Cana développement ou Champagne céréales développement) ou d'une filiale au cœur du métier de base (Sodiaal-Yoplait, voir paragraphe suivant).

\section{Exemple Sodiaal}

(produits laitiers, groupe coopératif national et international)

Union de coopératives avec partenaires financiers à $50 \%$ (PAI) sur la filiale (Yoplait).

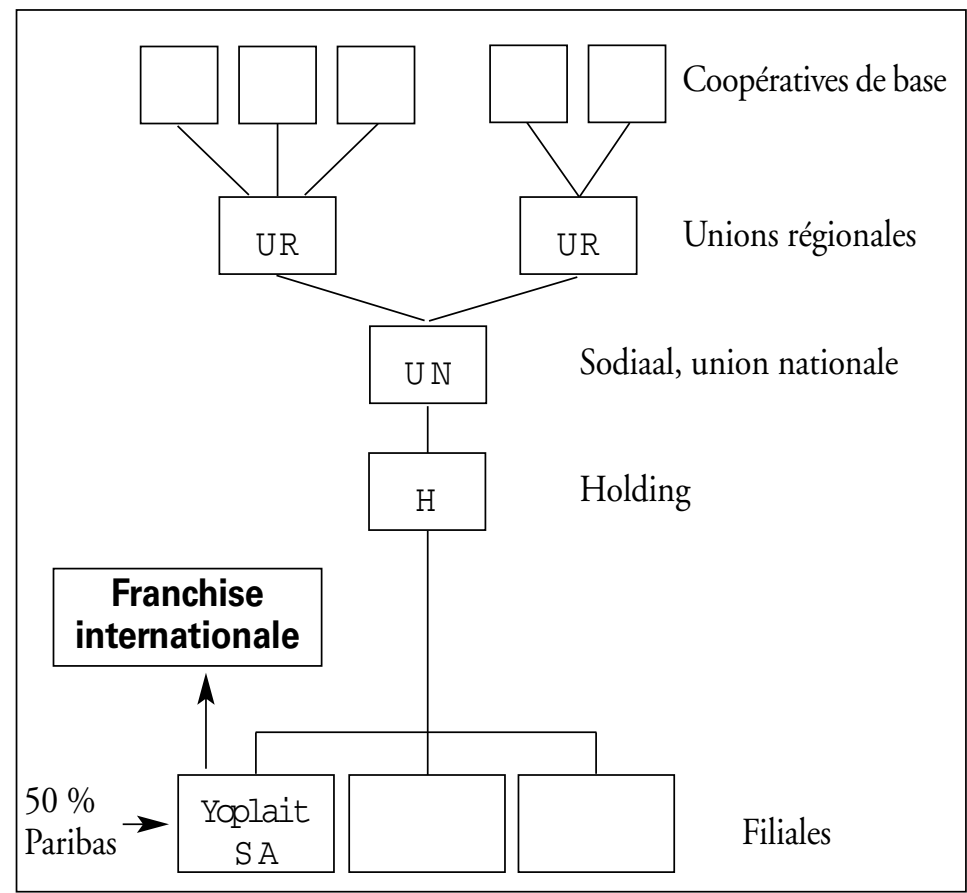

\section{Particularités}

- Union à plusieurs niveaux et filialisation des outils industriels et commerciaux depuis une dizaine d'années. 
- Stratégie internationale essentiellement par franchise de Yoplait, présence dans plusieurs dizaines de pays, numéro 2 mondial en produits frais laitiers derrière Danone.

- En 2002, suite à des difficultés financières et à des besoins de développement, participation de Paribas affaires industrielles (PAI) à hauteur de $50 \%$ dans la filiale la plus prestigieuse du groupe (Yoplait SA) avec la perspective d'une entrée en Bourse dans quelques années.

En plus des réflexions que nous avons conduites sur les autres cas, notamment en matière d'implications sur les modes de paiement des adhérents, il conviendra d'observer les conséquences en matière de décisions stratégiques.

En effet, dans le cadre de la globalisation des marchés et de l'internationalisation des concurrents (Danone et Nestlé), il serait sans doute pertinent pour PAI de pousser le développement international de la marque par une stratégie d'implantation industrielle, même si jusqu'à ce jour ce type de développement pour Yoplait n'a pas été couronné de succès (résultat positif pour la franchise, résultat négatif pour les implantations industrielles). Dans cette hypothèse, on assisterait, si la stratégie réussit, à une évolution de la finalité centrée jusqu'à ce jour sur la valeur de la coopérative pour l'usager, à un renforcement de la valeur du groupe coopératif pour l'actionnaire adhérent (possibilité de remontée des dividendes). On peut même penser que ce développement international entre en concurrence avec un développement en France qui, lui, pourrait apporter plus de valeur pour l'usager.

La pénétration en Bourse ne changerait pas significativement les données, si ce n'est la possibilité pour les adhérents en direct de prendre des actions Yoplait et la médiatisation plus importante du système. La stratégie pourrait bien sûr aussi dépendre de la nature des futurs actionnaires.

\section{Cas des coopératives de crédit et des mutuelles}

Ces transformations sont sensiblement plus avancées dans le secteur coopératif bancaire (Crédit agricole, Banques populaires) ou en mouvement dans le secteur mutualiste (rapprochement entre Groupama et Gan et perspective d'entrer en Bourse en 2005-2006).

Ainsi, un sociétaire du Crédit agricole peut être:

- sociétaire de sa caisse locale;

- porteur de CCI au niveau de sa caisse régionale (cotée en Bourse);

- actionnaire de Crédit agricole SA cotée en Bourse en 2002 (Casa).

Le secrétaire général du syndicat CGC du Crédit agricole, lors de la mise en Bourse, disait: "Le mutualisme n'avait qu'un seul but, la satisfaction des clients sociétaires par la qualité de notre service, le management et les équipes étaient tendus vers cet objectif. Désormais, ce n’est plus le client quil faut satisfaire, c'est l'actionnaire. C'est une véritable révolution culturelle ${ }^{(13)}$. "Ceci est d'autant plus perceptible que Casa est une filiale des caisses régionales qui va se comporter comme une "mère ". 
(14) Reprise des actifs de l'ULN par le groupe Bongrain il y a dix ans et aujourd'hui fusion de l'ULN avec la filiale de Bongrain (CLE).

(15) Voir article de René Mauget et Michel Forestier, Recma, n²79.
Dans les coopératives agricoles, les enjeux sont encore plus importants, car ce n'est pas seulement le taux d'intérêt ou la prime d'assurance qui est en jeu, mais l'essentiel du revenu de l'agriculteur (sa production).

\section{Conclusions sur les études de cas et implications sur les modes de rémunération des agriculteurs}

Ces différentes évolutions stratégiques et financières:

- délocalisation et internationalisation;

- rachat d'entreprises privées par la coopérative (apport de non-adhérents);

- partenariat industriel et commercial (partage du pouvoir et intérêts parfois divergents);

- partenariat financier (type PAI);

- cotation en Bourse de filiales;

- prise de participation des agriculteurs dans les filiales cotées ou non cotées;

- reprise d'entreprises coopératives par le secteur capitaliste ${ }^{(14)}$;

constituent les axes d'élaboration d'un nouveau paradigme pour les groupes coopératifs agro-alimentaires.

Les modèles antérieurs que nous avons évoqués ci-dessus n'expliquent plus cette nouvelle réalité. L'approche par le modèle de l'entreprise capitaliste centré sur l'apport de valeur pour l'actionnaire n'est pas non plus suffisante et pertinente. Le groupe coopératif constitue une nouvelle " race " d'organisation qu'il convient d'étudier. Une organisation complexe où la finalité consistera à optimiser sur le moyen terme et le long terme la double valeur pour l'adhérent: adhérent usager (apporteur de produit ou acheteur de produits ou de services) et adhérent actionnaire (dividendes et [ou] plus-value en fonction du capital), ceci en respectant les valeurs coopératives de la société mère et les valeurs citoyennes que l'on attend de toute entreprise, et notamment des coopératives qui l'affichent dans leur finalité et qui bénéficient encore de quelques avantages fiscaux.

En ce qui concerne la distribution du surplus, on peut distinguer trois cas de figure à partir des exemples précédents.

- Cas 1 : distribution peu différente de la coopérative classique, acompte + ristourne.

- Cas 2: prix de marché + dividendes avec avoir fiscal au prorata du capital social détenu par l'adhérent.

- Cas 3: prix de marché + dividendes via la coopérative + dividendes en direct de la filiale + perspectives de plus-values.

Remarque: un dosage entre ristournes et dividendes peut, bien sûr, être effectué dans la coopérative; de même, on peut doser avec la variable intérêt sur le capital social dans la coopérative, dans la limite des textes de loi en vigueur. Aux yeux des agriculteurs ${ }^{(15)}$, cette filialisation produit plusieurs effets:

- un effet d'éloignement souvent ;

- un effet de complexité (voir organigrammes présentés);

- un effet de brouillage de logique entre une logique coopérative pure et une logique capitaliste, brouillage qui a été accentué par la loi du 3 janvier 1991 avec la possibilité de remonter des dividendes jusqu'aux 
agriculteurs via la coopérative (cas 2) et la prise de participation directe dans les filiales (cas 3).

\section{A la recherche d'un renouveau coopératif au XXl ${ }^{e}$ siècle}

Depuis 1966 et la recommandation 127 de l'Organisation internationale du travail (OIT), la coopérative n'est plus simple association de personnes. Elle devient entreprise. Elle le restera dans les révisions en cours des textes qui la définissent, car l'idée d'entreprise est le fruit d'une lente maturation autour d'un difficile consensus universel, initié par l'Alliance coopérative internationale $(\mathrm{ACI})$, en période de guerre froide Est-Ouest et de décolonisations Nord-Sud.

Entreprise, le métier de la coopérative n’est alors plus de transformer la société, mais de promouvoir les bonnes pratiques de gouvernance, le bon modèle coopératif de gestion.

Ainsi, on ne se trompera pas de cible et l'on évitera de développer un coopérato-centrisme dépassé, encore présent dans la seconde moitié du siècle précédent, avant l'effondrement de toute tentation de deuxième voie et, partant, de troisième voie coopérative.

Dépouillée de ses ambitions, la coopérative est néanmoins immergée dans la société globale, dans un environnement total aux différents étages de sa prégnance, du « local », ou territorial - aujourd'hui à la mode -, jusqu’à l'universel de la "mondialisation ", autre extrême également débattu.

A sa juste place, la coopérative participe à l'interface. Elle induit des mutations dans la société par sa force de proposition économique et sociale, mais elle est elle-même soumise aux lois, contraintes et opportunités de cette même société (idée de "process » continu de la socio-économie).

A sa juste place, mais mieux que les autres entreprises, la coopérative inclut dans ses obligations initiales la préoccupation d'une bonne relation à l'environnement. Même si elle provoque controverse, la règle existe (OIT, 1966) et ne demande quà être travaillée.

Par sa présence et son intervention, la coopérative assure une intermédiation plus ou moins réussie entre les publics qu'elle représente légitimement et les politiques-décideurs à leurs différents échelons de responsabilité. Quels sont ces décideurs et leurs intérêts différents? Quelles sont les approches de convergence aujourd'hui connues? Deux points à développer maintenant.

\section{Pluralité de décideurs, confusion d'intérêts}

Les acteurs sont aujourd'hui plus nombreux, avec l'admission de nouveaux entrants dans la coopérative de base et dans le groupe. Il s'agit essentiellement des forces financières externes dont la coopérative a besoin pour poursuivre son développement en atteignant les dimensions requises par la compétition mondiale. La notion juridique d' associés non coopérateurs " rend bien mal compte de la réalité économique, qui est celle de partenaires 
(16) Serge Koulytchizky, «Le qua drilatère $d^{\prime}$ Henri Desroche revi sité », Recma, n 272, avril 1999. sollicités, confortés par les liens contractuels tissés autour de holdings protecteurs, qui est aussi celle des financeurs en direct des filiales.

En revisitant le quadrilatère d'Henri Desroche ${ }^{(16)}$, nous avons montré la dimension nouvelle qu'ont prise dans le schéma les acteurs externes, pour deux raisons:

- l'extension des marchés du fait de la mondialisation, de la globalisation, du progrès des outils de la communication;

- la délégation aux unités de base (subsidiarité) des différentes missions de reconstruction du tissu économique et social, que ne peuvent plus accomplir les Etats fédérés.

Il ne s’agit plus alors de se référer à un " environnement » indifférencié; il faut classer les acteurs nouveaux.

La tâche est ardue, plus ardue encore si nous traitons ici non pas de la coopérative basique, mais du groupe à formes multiples et à têtes de décision - outre la mère, les filiales - nombreuses, même si les autonomies apparaissent « limitées » en droit.

L'énumération des acteurs internes et externes qui interviennent dans la décision coopérative est abordée dans l'article cité sur le quadrilatère revisité. Elle est développée plus longuement, sous forme de représentation typologique, dans la contribution Addes 2002; elle sera reprise dans un cahier spécial Maraussan ("Les cahiers de l'économie sociale ", $\mathrm{n}^{\circ} 2$, à parâtre).

Lintervention est tantôt active, tantôt passive, du fait de la seule existence de l'acteur (... la peur du gendarme régalien).

Avec la notion de "parties prenantes ", on voit poindre l'approche par «stake-holders » anglo-saxonne, ici très largement et très coopérativement interprétée. Ne sont plus retenus uniquement les fournisseurs et acheteurs, mais tous les partenaires du territoire de la coopérative, de sa zone d'implication ("périmètre de solidarité " selon L. Monnier), qui pèsent sur les décisions prises en assemblée générale, directement ou indirectement. Alliés certes, mais aussi élus politiques locaux, syndicats... et généralement tous « intéressés " qui sont en attente d'emploi, de contrat, de réaction aux décisions et anticipations de la coopérative. C'est là le principal du jeu de l'intermédiation coopérative.

La notion nouvelle de " parties prégnantes » indique le caractère incontournable dans les choix de forces et de réseaux externes. Les uns porteurs de connivences amicales: pour donner un seul exemple, fédérations, confédérations, nationales, européennes, mondiales, liées au champ coopératif ou professionnel considéré, à l'intercoopération, au devenir du monde paysan, à l'agriculture raisonnée, au développement durable... Les autres chargés de forces inamicales: pour donner un exemple très général, Davos est amical à Davos et inamical à São Paulo, São Paulo est amical à São Paulo, inamical à Davos...

Pour les deux catégories de parties " prégnantes ", on peut encore les classer comme agissant:

- avec lien de sujétion ou sans lien de sujétion;

- avec lien de contractualisation, sans lien contractuel.

La dichotomie acteurs endocentrés-acteurs exocentrés renforce la bijection 
d'objectifs dans le projet coopératif. D'un côté, une logique de résultats portée par l'équipe managériale appuyée par les nouveaux entrants plus ou moins proches du "modèle d'affaire "; de l'autre, une logique de redistribution dont sont porteurs les élus avec désormais l'appui curieux mais logique des salariés au prorata de leurs salaires, stock-options, attributions de parts privilégiées.

$\begin{array}{lll}\begin{array}{l}\text { Manageurs }+ \text { financeurs } \\ \text { logique d'investissement } \\ \text { (long terme) }\end{array} & \neq & \begin{array}{l}\text { Elus + salariés } \\ \text { logique de redistribution } \\ \text { (tout, tout de suite) }\end{array}\end{array}$

On notera la position désormais écartelée des dirigeants salariés, à la fois intéressés au développement coopératif par accumulation et investissement - question d'image de marque de cadres compétents - et intéressés par la distribution rapide des résultats - pour ne pas dire du profit - afin de ne pas risquer les aléas du « jeu de culbuto » des stock-options et parts sociales. Cette tendance n'est pas propre à l'univers coopératif, mais elle renforce la question de l'endocentration ou de l'exocentration de certaines catégories d'acteurs internes.

Elle apparaitra encore plus inquiétante dans le groupe coopératif avec la tentation des distributions de dividendes de filiales et de rentes boursières, sil y a entrée en Bourse.

Qui est désormais le plus proche du cœur d'entreprise coopérative: le groupe des manageurs-financeurs intéressés au développement de la « firme » ou le groupe sociétaires-élus parfois tentés par une économie de rente? La question est dérangeante, mais doit être posée.

\section{Convergence d'approches ou grand écart}

La seconde moitié du XXe siècle a peu à peu fait apparâtre dans le groupe coopératif une bijection de plus en plus affirmée entre une logique de résultats portée par l'équipe managériale (technostructure salariée) et une logique de redistribution dont sont porteurs les politiques (élus bénévoles).

La tradition anglo-saxonne supporte dans le domaine de la gestion la quasitotalité des connaissances acquises en Europe sur le modèle des grandes écoles américaines et de leurs abondantes publications.

Dans le champ des coopératives et plus généralement des organisations à but non lucratif (NPO), cela se traduit par une présentation duale qui sépare nettement la gestion coopérative appartenant aux sociétaires dans leur assemblée générale et aux administrateurs élus, d'une corporate governance dévolue aux agents désignés par les premiers.

Exprimé ainsi, le rôle premier paraît revenir à la catégorie des sociétaires; dans la réalité, nous trouvons d'un côté une prise de décision quasi autonome des agents qui fonctionnent en continu, alors que les sociétaires n'ont qu'un pouvoir de "votation " séquentiel qui s'exprime une fois l'an sur des bilans complexes et des projets préparés par la technostructure. 
Au mieux, un "board " réunira les têtes des deux pyramides, directeur général et président du CA. Cette modalité de cogestion peut être organisée dans un organigramme. Elle est indispensable, fruit de l'adaptation progressive naturelle des structures d'entreprise aux décisions à prendre dans l'urgence ( $80 \%$ de l'activité du chef d'entreprise, selon $\mathrm{H}$. Mintzberg), qui requièrent la présence constante et concomitante des deux signataires.

Cependant, cette cogestion de fait, typique de la méga-coopérative, crée une nouvelle dissociation, cette fois non plus verticale, entre exécutif et législatif, mais horizontale entre administrants et administrés; on pourrait aussi dire " gouvernants » et " gouvernés ".

A ce phénomène de basculement déjà ancien et connu, le pouvoir de décider passant peu à peu des élus à la classe managériale et dans la méga-coopérative aux sommets associés (gouvernants), s'ajoute, avec la croissance de dimension des entités coopératives, l'irruption dans le schéma de nouveaux entrants exocentrés; nous voulons parler de capitalistes, personnes physiques ou morales désormais associées au groupe-holding.

Certes, la méga-firme a besoin de capitaux pour conforter sa croissance externe. Cela induit bien évidemment des raisonnements différents entre l'univers des valeurs d'hier et celui actuel de l'efficacité et même du profit, une bijection dans le projet; cela induit encore une mixité des structures, au mieux du type de la cogestion, au pire une contre-gestion si aucune mixité ne se trouve opérante et si aucune procédure d'arbitrage ne fonctionne.

Le groupe coopératif agricole moderne se trouve placé à ce carrefour.

Il peut y avoir, comme dans toute autre firme, des ajustements, des recentrages sur le métier initial, abandon de telle ou telle source de profit non avérée, mais tout cela est stratégie ordinaire, pas spécialement coopérative.

Les adhérents coopérateurs de base se trouvent " saisis » dans cette construction. Ils sont désormais impliqués au titre d'une triple ou quadruple qualité: propriétaires associés, usagers contractuels (un ou deux degrés avec les contrats d'intégration), associés actionnaires de la coopérative ou des filiales, actionnaires dans le mécanisme boursier plus ou moins joueurs en direct ou par délégation selon les conventions de départ (pactes d'actionnaires).

Désormais, avec les moyens nouveaux de communication, avec les sources multiples d'information - dont audits et éventuellement cotations externes -, la coopérative entre dans un système de comparaison tous azimuts, au même titre que l'entreprise de capitaux. Cela exige d'elle une transparence à laquelle elle n'était pas accoutumée. Le " consensus agricole ", pratiqué jusque-là, était d'autant plus généralisé que l'information restait asymétrique.

Si l'on veut éviter le grand écart entre financeurs et sociétaires, entre technostructures et bases paysannes, entre accumulation et redistribution de revenus agricoles, il faut souhaiter l'harmonisation du « discours ».

Mais cette convergence ne peut que rester imparfaite dans la mesure où les finalités ne sont pas les mêmes, profit-rentabilité d'un côté, utilité socialeefficacité de l'autre.

Cependant, le vocabulaire peut faire illusion. Légitimité, loyauté, équité, transparence, local peuvent faire partie du discours coopératif (Maraussan) 
(17) Groupe de travail du Ciriec intemational. Publication 2001, De Boeck-Université, Bruxelles.

(18) Daniel Côté ajoute " [...] Cette hypothèse ne fait que reprendre l'analyse des tendances lourdes que nous avons identifiées précédemment. Sur la base de cette hypothèse, nous pouvons anticiper un affaiblissement dans l'intensité d'application des règles coopératives, alors que les règles $d u$ marché s'intensifient [...] Lors de la création de la coopérative, la fonction entrepreuneuriale est forcément assurée par les membres (forte intensité d'application des règles coopératives). De plus, dans la mesure où le contexte initial est régulièrement associé à diverses situations d'abus, nous croyons pertinent de parler d'un environnement faiblement concurrentiel (monopole, absence de marché solvable, asymétrie d'information, etc.)." comme du discours attaché à la théorie de la firme, mais il faut prendre garde aux "faux amis ", aux termes dits " déceptifs ".

La légitimité historique et patrimoniale coopérative et la légitimité par les seuls droits de propriété des actionnaires ne sont pas une seule et même chose. La loyauté coopérative, qualité et transparence, n’est pas la seule « fidélisation » des clients et fournisseurs que vise le business model.

Le " juste prix » comporte une dimension équitable et solidaire qui ne figure pas dans le modèle anglo-saxon.

Il faudrait un sérieux toilettage du vocabulaire si l'on voulait avoir un langage commun, une même vision des standards et des bonnes pratiques de la gouvernance d'entreprise.

\section{Performance globale en mouvement}

A la fin de l'ouvrage collectif sur les holdings coopératifs déjà cité, Daniel Côté présente une schématisation des conclusions tirées des travaux d'une trentaine de chercheurs originaires de quatorze pays qui se sont déroulés sur une période de trois ans ${ }^{(17)}$. Il présente la figure suivante (figure 1a) et stipule que " toutes choses égales par ailleurs nous assistons à un glissement de la position A (1 ${ }^{\text {er }}$ quadrant) à la position B (3e quadrant) " ${ }^{(18)}$.

Cependant, le modèle s'appuie sur une convention de représentation: "Les coopératives émergent dans le quadrant I; la possibilité de création dans les quadrants II et IV est admise; en III elle est exclue. "

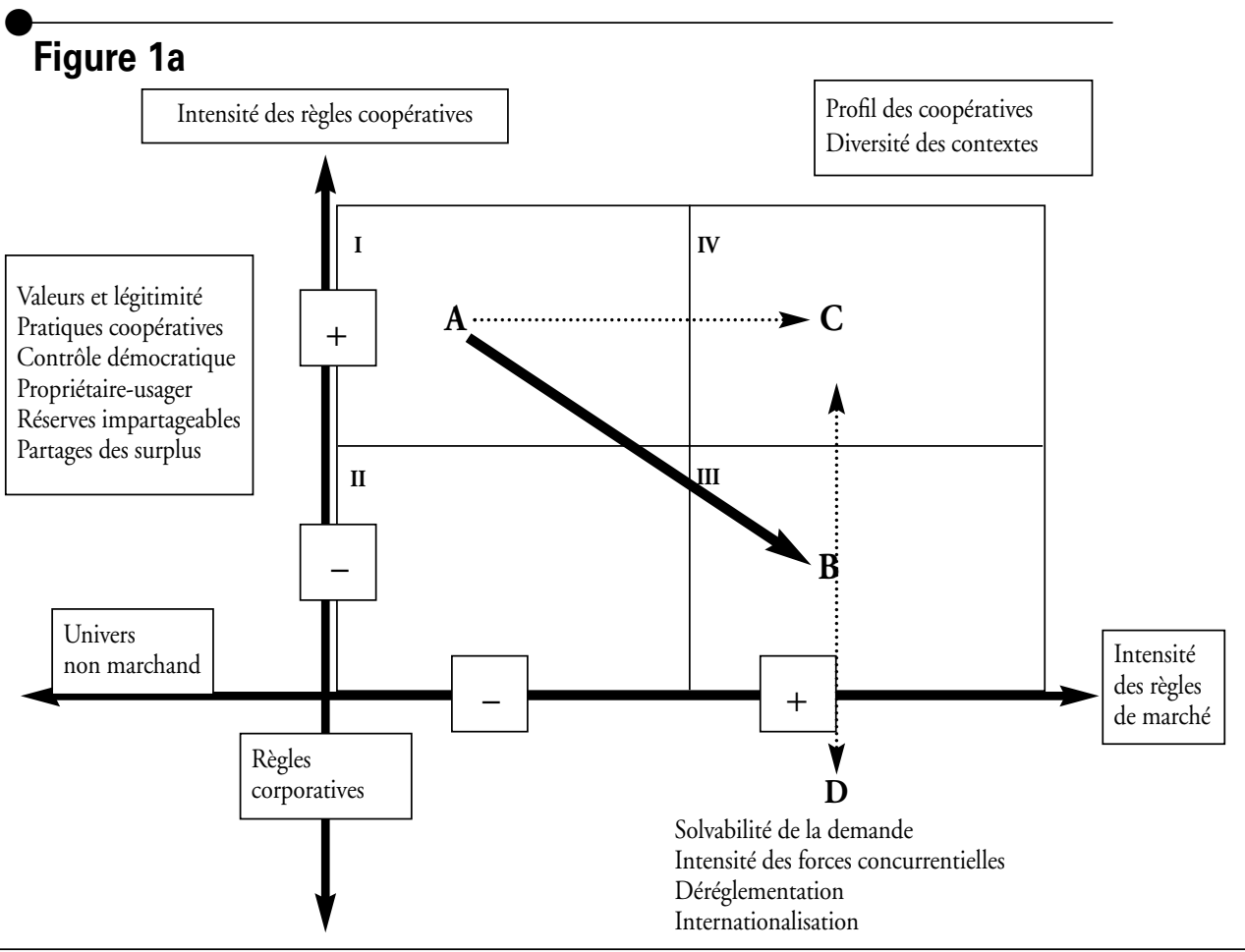


(19) Ouvrage diffusé à Naples, septembre 2002 (Ciriec interna tional éditeur).
Nous apportons ici, en complémentarité (figure 1b), une forme plus universelle de voir les choses, avec des axes différents et un point de départ situé à l'origine des deux axes (en 11), correspondant à la coopérative « en apprentissage », ni totalement formée à une vaste solidarité, ni totalement capable en matière de gestion efficace. La motivation sera ici celle de la recherche de synergies entre un petit nombre de coopérateurs solidaires qui veulent améliorer leur situation économique et sociale face aux abus des négociants. Héritée des pratiques traditionnelles de l'entraide, cette position sera celle de la majorité de nos coopératives françaises en émergence.

Entre la voie de l'utopie et celle du parfait pragmatisme marchand, la nouveauté de l'approche réside dans l'ambition de tracer un « chemin coopératif vertueux ", voie d'un nouveau paradigme coopératif, si l'on veut l'appeler ainsi. Il repose sur le concept de performance globale de l'entreprise, terme emprunté au XI plan (commission innovation, président J. Gandois).

Ici nous parlerons:

- de performance en solidarité (axe des ordonnées);

- de performance de position sur les marchés (axe des abscisses).

Si l'analyse de ce dernier axe est couramment pratiquée avec des outils de diagnostic connus, l'axe des solidarités demande explication.

Si l'on admet provisoirement la convergence entre notion d'intérêt général et notion de solidarité, nous pouvons nous référer à B. Enjolras, qui écrit dans Economie plurielle et régulation socio-économique ${ }^{(19)}$ : "Lintérêt général est un construit social, ce qui implique qu'il n'existe pas une conception donnée de ce qu'est l'intérêt général, mais que les conceptions varient dans le temps, l'espace et au travers des cultures; l'intérêt général résulte de l'effet externe d'une activité économique, externalité qui affecte la totalité des membres d'une communauté quelle que soit l'échelle de cette communauté: locale, régionale, nationale, supranationale. " Ce postulat est le premier résultat des recherches d'un groupe de travail du Ciriec international qui va poursuivre ses travaux sur le sujet.

Notons que les indicateurs sociaux de J. Delors, les différentes étapes des bilans sociaux d'entreprise préfigurent déjà la recherche d'un bilan sociétal qui permettra sans doute des internalisations nouvelles sur l'axe des solidarités. Déjà la CFCA travaille à l'expérimentation d'un tel bilan pour les coopératives agricoles en cinq domaines et près de cinq cents indicateurs (démocratie-vie interne de la coopérative, promotion des hommes, solidarité-mutualisme, performance économique, action sur le territoire).

Notre nouvelle matrice $1 \mathrm{~b}$ est de type graphique en nuages.

- Elle permet de placer aux différentes positions repérées par la numérotation la totalité des cas présentés dans la première partie de cet article et ceux - étrangers pour la plupart - qui figurent dans l'ouvrage sur les holdings coopératifs. Elle a été construite à partir de l'analyse de multiples expériences recueillies par nos étudiants.

- Elle permet encore de suivre des trajectoires coopératives.

Ainsi en est-il pour l'Union laitière normande (ULN) de sa fondation, au lendemain de la guerre $(31,32)$, à la privatisation en fin de siècle $(12,13$, puis sortie du cadre). 


\section{Figure $1 \mathrm{~b}$ - Performance globale d'entreprise coopérative}

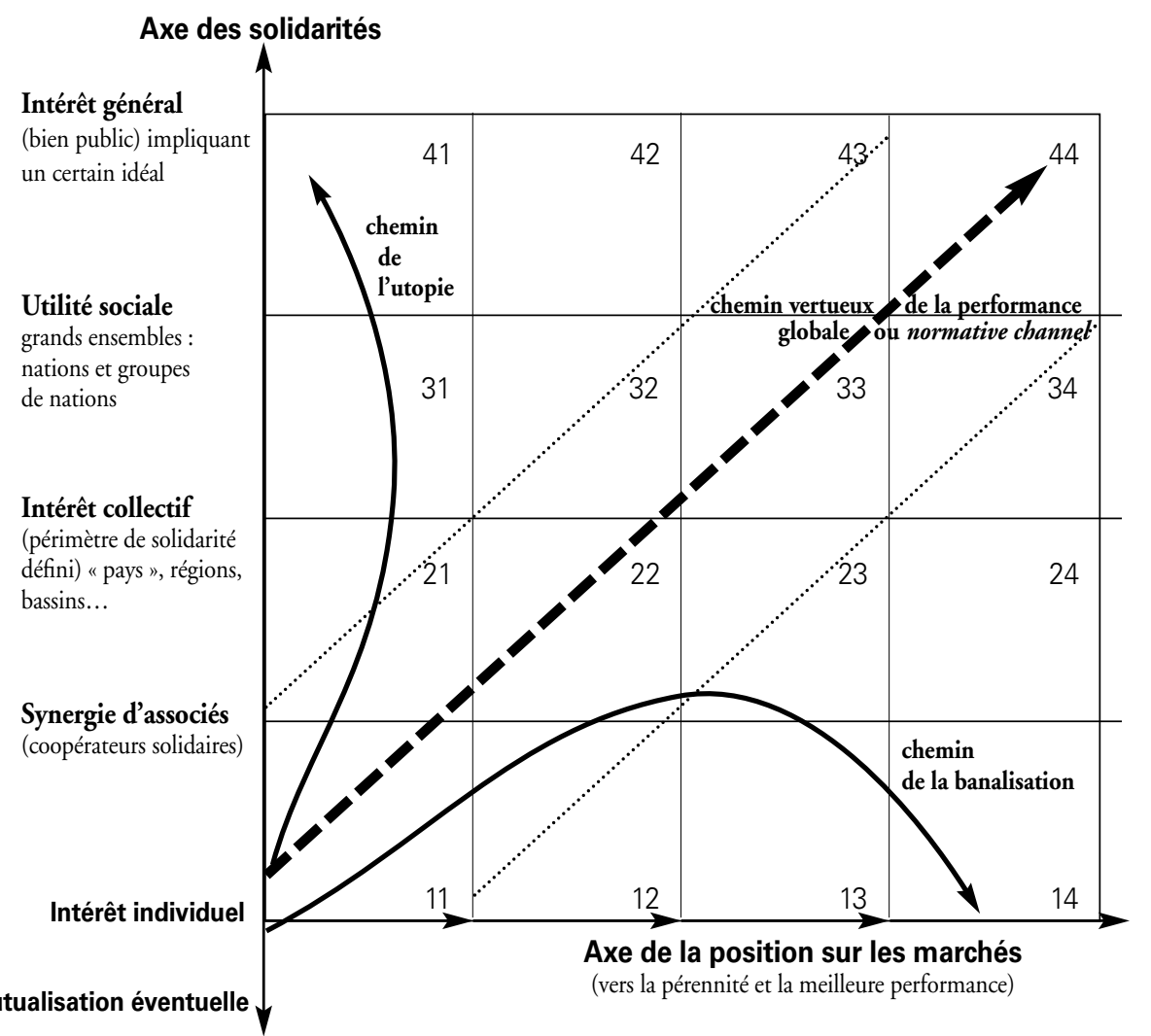

11. Coopérative en apprentissage, peut vivre temporairement dans une niche: essaimage dons, subventions des alliés et des réseaux amis.

41. Absence d'efficacité d'entreprise confinmée, repose sur la philanthropie ou sur les subventions publiques, risques de disparition.

14. Leader économique en risque de banalisation, voire de démutualisation.

44. A la fois leader économique et parfaite implication dans la solidarité la plus vaste.

Entre les pointillés : zone de danger de basculement d'un côté ou de 1'autre.

Chemin « vertueux » si l'on se réfère à un système de valeurs de niveau supérieur (Aristote).

"Normative channel " si l'on s'en tient au calcul de la meilleure voie possible (L. P. Bucklin).

Ainsi en est-il pour les Cuma, les unes opportunistes (11 à 14), les autres utopistes (11 à 41) ou encore vertueuses (11 à 44).

Ainsi encore pour le biologique coopératif, de l'utopie (Biocoop) à la prégnance des marchés (Biocap). Même chose pour le commerce éthique (M. Havelaar).

- Elle permet de repérer la position d'une coopérative par rapport aux nuages dominants du moment, du cœur de métier, de la zone de développement et à l'évolution de ces nuages dans le temps.

- Elle permet enfin de percevoir des convergences insoupçonnées avec des formes d'entreprises, autres que coopératives, qui œuvrent sur le même axe de solidarités: régies publiques, mécénat d'entreprise, entreprises « citoyennes " ou appartenant aux secteurs mixtes, et paritaires de l'économie. En parallèle, l'axe coûts-performance marchés doit être également analysé. 
Ainsi apparaissent d'autres dimensions, qui sans être explicites se lisent facilement à l'analyse du graphique:

- le temps, évolution d'une unité et évolution d'un groupe d'activité;

- l'espace, par zones de développement et niveaux;

- le statut, coopératif, d'économie sociale et autres permettant tous comparatifs.

Le modèle permet d'établir dans la durée la corrélation entre solidarité coopérative et efficacité d'entreprise.

La leçon principale d'un paradigme de bonne évidence, qui a été soumis à expérimentations multiples, pourrait se résumer par l'adage suivant:

"Pas de vertu coopérative sans bonnes affaires... Pas de bonnes affaires, pour la coopérative, sans vertu."

Bien sûr, nous ouvrons ainsi le champ à de multiples commentaires, à de multiples interrogations sur ce qui est ou non "vertu ", sur ce qui est ou non «bonnes affaires " (good business); qu'est-ce que l'un des deux volets du propos apporte à l'autre et inversement - toutes choses égales par ailleurs -, c'est-à-dire à l'intérieur du système économique mondial qui nous régit actuellement (la pensée dite " unique »)?

La question principale est alors celle-ci: quest-ce qui peut maintenir la coopérative à proximité de l'axe vertueux et ramener à la raison les unités qui ségareraient?

C'est affaire de régulation, de principe ou d'existence d'un régulateur.

Nous ouvrons là un débat de société, qui inclut une dimension politique (de science politique) et déborde largement le cadre de l'entreprise. Il concerne au premier chefl'ACI, véritable parlement de l'économie coopérative. L'alliance est confrontée à cette interrogation: "Qu'est-ce qui fait sens dans la coopérative aujourd'hui? » comme le demande J. Grave en France; même attente de renouveau au Québec, à l'instigation de la caisse Desjardins. Une recherche est actuellement en cours en partenariat avec D. Côté.

\begin{tabular}{|c|c|c|c|c|}
\hline Pays & $\%$ Agri/UE & $\%$ IAA/UE & $\%$ Coop/UE & Niveau coop ${ }^{(1)}$ \\
\hline & 1998 & 1999 & 1998 ou 1999 & \\
\hline Allemagne & 15 & 20 & 17 & + \\
\hline Autriche & 1,6 & 2,2 & $?$ & \\
\hline Belgique + Luxembourg & 3 & 3,6 & 1,6 & - \\
\hline Danemark & 2,9 & 2,7 & 5,3 & ++ \\
\hline Espagne & 12,4 & 9,5 & 4 & - \\
\hline Finlande & 1 & 1,4 & 4 & ++ \\
\hline France & 21,6 & 21,3 & 30 & + \\
\hline Grèce & 4,1 & 0,9 & 1,4 & - \\
\hline Irlande & 2 & 2,6 & 5,2 & ++ \\
\hline Italie & 16 & 10,4 & 8 & - \\
\hline Pays-Bas & 5,7 & 6 & 10,7 & ++ \\
\hline Portugal & 1,8 & 1,7 & 0,4 & - \\
\hline Royaume-Uni & 8,3 & 15,3 & 5,8 & - \\
\hline Suède & 1,5 & 2,6 & 4,6 & ++ \\
\hline Total UE & $\begin{array}{c}100 \% \text { soit } \\
213 \text { milliards d'euros }\end{array}$ & $\begin{array}{c}100 \% \text { soit } \\
587 \text { milliards d'euros }\end{array}$ & $\begin{array}{c}100 \% \text { soit } \\
215 \text { milliards d'euros }\end{array}$ & \\
\hline
\end{tabular}




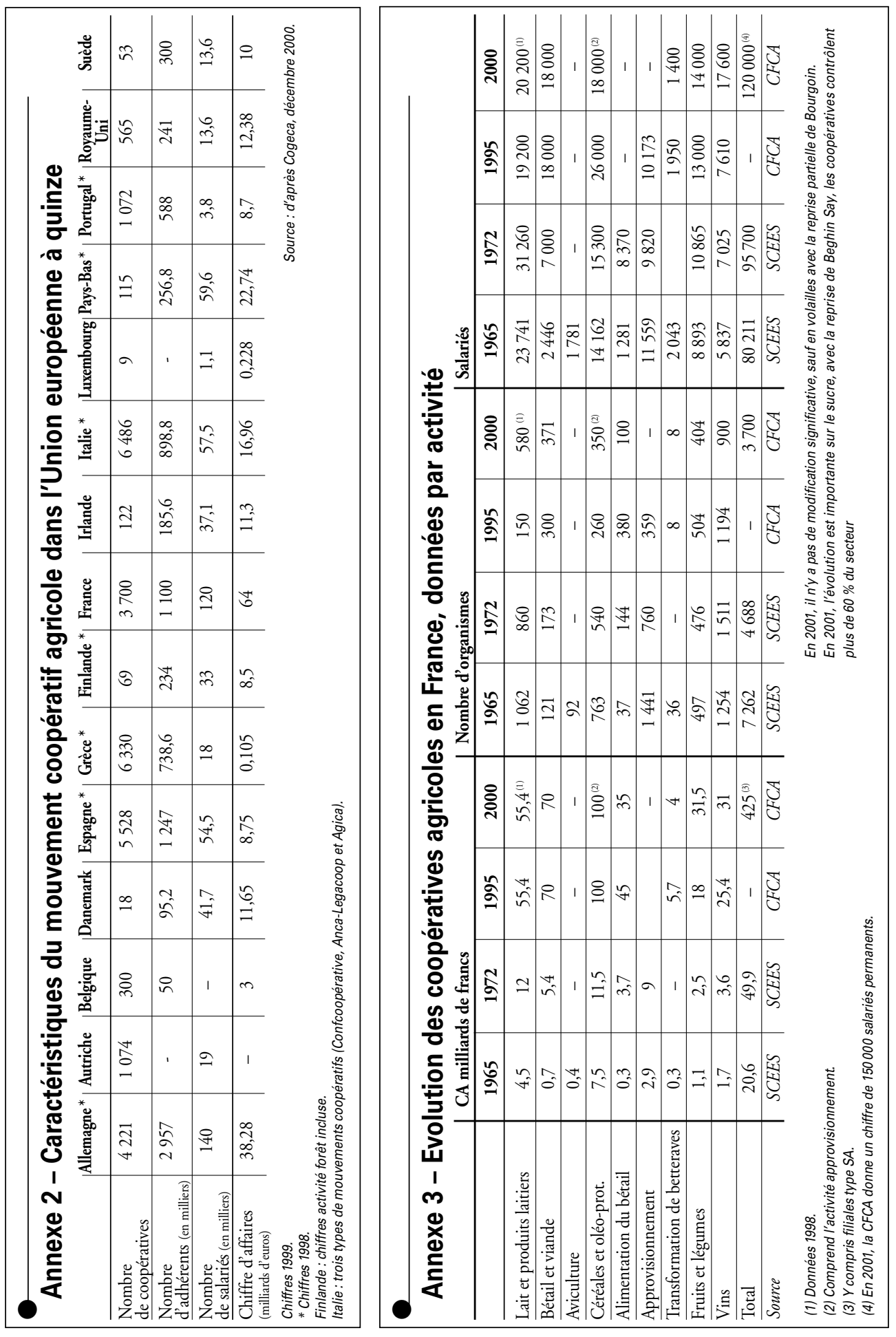




\section{Annexe 5 \\ Sigles utilisés}

ACI: $\quad$ Alliance coopérative internationale.

Addes: Association pour le développement de la documentation en économie sociale.

BIT: Bureau international du travail.

CA: chiffre d'affaires.

CAF: capacité d'autofinancement.

CCI: $\quad$ certificats coopératifs d'investissement.

CFCA: Confédération française de la coopération agricole.

CIA: $\quad$ Confédération internationale de l'agriculture.

Ciriec: Centre international de recherche et d'information sur l'économie publique sociale et coopérative.

CNMCCA: Confédération nationale de la mutualité, du crédit et de coopération agricole.

Cogeca: Comité général européen de la coopération agricole.

Cuma: coopératives d'utilisation de matériel agricole.

EBE: excédent brut d'exploitation.

IAA: industries agro-alimentaires.

Jac: Jeunesse agricole catholique.

OIT: Organisation internationale du travail.

Pac: politique agricole commune.

PAI: Paribas affaires industriels.

SCEES: Service central des études économiques et statistiques.

Sica: $\quad$ société d'intérêt collectif agricole. 


\section{Bibliographie sélective}

Holdings coopératifs, ouvrage collectif Ciriec, De Boeck éd., Bruxelles, 2001.

"Les cahiers de l'économie sociale ", $\mathrm{n}^{\circ} 2$, dossier colloque Maraussan 2001 (à paraître).

Dossier colloque Addes, octobre 2002, « Le développement des groupes coopératifs depuis un demi-siècle », $65 \mathrm{p}$.

"Les cahiers de l'économie sociale ", $\mathrm{n}^{\circ} 1$, Coopération et économie sociale au second XX' siècle, Claude Vienney (1929-2001), Institut de l'économie sociale-L'Harmattan, Paris, juillet 2002. Jacques Bertelot, Les coopératives agricoles en économie concurrentielle, Cujas, 1972

J. Caby et G. Hirigoyen, La création de valeur de l'entreprise, Economica, 1997.

Daniel Côté (HEC Montréal), « Les coopératives et le prochain millénaire: l'émergence d'un nouveau paradigme ", Recma, n 275-276.

Demoustier Danièle, L'économie sociale et solidaire, Syros, 2001.

Gérard Deshayes, Logique de la coopération et gestion des coopératives, Skippers, 1988.

Gérard Deshayes, "La société de capitaux, la coopérative, les entreprises de l'économie sociale et la théorie de l'agence ".

Serge Koulytchizky, "Nouveaux instruments d'analyse des coopératives ", Revue des études coopératives, ns 199 et 201, 1980 (50 p.).

Serge Koulytchizky, "Le quadrilatère d'Henri Desroche revisité ", Recma, n² 272, 1999 (12 p.).

René Mauget, "Quelles stratégies pour les coopératives agricoles?", thèse Paris I, 1982.
René Mauget, Evolution des coopératives durant les trente dernières années, approche statistique et stratégique: l'entreprise coopérative, expérience et recherche francophone, Pulim, 1998.

René Mauget, " Les moyens de financement des groupes coopératifs agro-alimentaires et leurs conséquences ", Economie et Gestion agroalimentaire, octobre 1991.

René Mauget et Francis Declerck, " L'analyse stratégique des groupes coopératifs européens ", Economie et Gestion agro-alimentaire, octobre 1993.

René Mauget, "Le partenariat coopérativesfirmes privées ", Economie et Gestion agroalimentaire, avril 1994.

René Mauget et Michel Forestier, colloque Addes 2000 "De la coopérative au groupe coopératif, évolution ou dénaturation? " et Recma, $\mathrm{n}^{\circ}$ 278-279.

René Mauget, "La course à la taille », Euralis info, décembre 1999.

René Mauget, "Les coopératives agroalimentaires face à la globalisation des marchés ", colloque Sfer, juin 2000.

Philippe Nicolas, « La formation des groupes coopératifs agro-alimentaires et la situation des agriculteurs sociétaires ", Economie et Gestion agro-alimentaire, juillet 1990.

Raymond P., Coopération agricole, combat pour l'unité, Pays d'ici et d'ailleurs, 1979.

Claude Vienney, Socio-économie des organisations coopératives, tome II, 1982. 